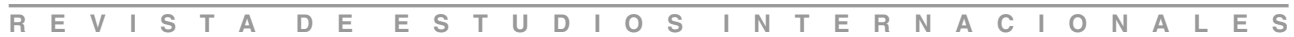

\title{
Relación de la Unión Europea con América Latina. Madrid y después
}

\author{
Dra. Amalia Stuhldreher
}

El fin del siglo XX fue señalado por el cambio radical del sistema internacional. La superación de la confrontación bipolar determinaría el surgimiento de un orden unipolar en el área militar de la arena internacional y de un orden multipolar en los ejes político-diplomático y geoeconómico. El fortalecimiento de los procesos de globalización, sobre todo el fuerte impulso de los mercados financieros internacionales, la orientación primigenia de la producción hacia los mercados globales, así como el aumento de las corrientes de inversión pasarían a ser los rasgos centrales de la economía internacional. En dicho contexto, los procesos de integración regionales alcanzarían nueva y creciente importancia. Lo mismo sucedería con las relaciones interregionales, esto es, las relaciones entre los diferentes bloques económicos debido a su potencial tanto de cooperación como de conflicto, ya que el alto grado de interdependencia de las economía nacionales determina que sólo en casos excepcionales estos fenómenos se limiten al campo nacional o regional. Por el contrario, en numerosas ocasiones tanto la dinámica de cooperación como de conflicto tienden a generar efectos de derrame, que se extienden a través de las regiones hasta alcanzar el campo internacional ${ }^{1}$.

Así pues, en un contexto global en el que las dimensiones económicas y comerciales se estructurarían crecientemente como un orden regulado de bloques económicos ${ }^{2}$, las relaciones de la Unión Europea (UE) con otros bloques regionales adquirirían nueva relevancia en función de su potencial económico, su carácter ejemplar para otras estructuras regionales, y debido a las connotaciones implícitas para el campo internacional. En ese sentido, la discusión acerca del modo de relacionamiento interregional de Europa occidental se vinculó con frecuencia con el debate en torno a la calidad del actor UE en el sistema internacional.

${ }^{1}$ Véase Norman Palmer. "The New Interregionalism in Asia and the Pacific", Massachussetts/Toronto, Lexington Books, 1991, pág. 116.

${ }^{2}$ Véase Elmar Altvater / Birgit Mahnkopf. "Grenzen der Globalisierung: Ökonomie, Ökologie und Politik in der Weltgesellschaft”, Verlag Westfälisches Dampfboot, Münster, 1999. págs. 388-389. 
INTRODUCCIÓN: LA POLÍTICA INTERNA-

CIONAL EN UN CONTEXTO DE CAMBIO

RADICAL

1 presente análisis comprende cinco secciones: en la primera se sistematizan algunas consideraciones teóricas respecto del rol general de la UE como actor internacional. En la segunda, se examina la dimensión comercial de la política exterior comunitaria, dada su repercusión en la conformación de los vínculos con América Latina. La sección siguiente presenta el cuadro general del relacionamiento de la UE con América Latina, con énfasis en los ejes considerados clave por los latinoamericanos, esto es, las relaciones económicas y la cooperación internacional. La cuarta sección centra la atención en los resultados de la Cumbre UE-Latinoamérica/Caribe, realizada en Madrid en mayo de 2002, y se refiere a los diversos matices y ritmos de avance de las negociaciones con los grupos subregionales. Finalmente, se efectúan algunas reflexiones respecto del verdadero sentido y alcance de la así llamada relación estratégica UE-América Latina/Caribe.

\section{LA UE EN SU CALIDAD DE ACTOR INTERNACIONAL EN UN MUNDO \\ GLOBALIZADO}

En relación con el debate acerca del rol de la UE en el campo internacional, en las relaciones internacionales se dieron paradigmas interpretativos alternativos en la forma de modelos realistas e idealistas, que impregnaron de manera decisiva la discusión: por una parte, la primera corriente subrayaría el rol aún determinante de los Estados nacionales en la concepción e implementación de la política exterior de Europa, aun cuando advirtiera expresamente el carácter tangencial de las nuevas tendencias en este ámbito. Así, aunque en esta perspectiva se subrayara la dimensión nacional como el núcleo todavía central de la diplomacia europea, no se dejaría de señalar la existencia de corrientes 'nacionales' y 'colectivas' en una situación de 'equilibrio dinámico's ${ }^{3}$. Por otra parte, las interpretaciones idealistas enfatizarían las instancias supranacionales y el rol de la UE como 'poder civil' o 'civilizador', en contraposición a otros grandes poderes como los Estados Unidos, cuyas decisiones de política exterior estarían mayoritariamente determinadas por sus propios intereses y por consideraciones de hegemonía. Para la UE existiría la posibilidad de asumir responsabilidad a ni-

\footnotetext{
${ }^{3}$ Véase Christopher Hill. "The Actors Involved: National Perspectives”, en Elfriede Regelsbeger / Philippe de Schoutheete de Tervarent / Wolfgang Wessels (eds.). Foreign Policy of the European Union. From EPC to CSPC and Beyond, Lyenne Rienner Publishers, Boulder, Londres, 1997, págs. 85-97.

${ }^{4}$ Los conceptos de civilising power y civilian power fueron introducidos por Christopher Hill. Véase al respecto: Christopher Hill, "European Political Cooperation Considerd as Foreign Policy", Conference Paper, British International Studies Association, 1983.
} 
vel internacional mediante su compromiso activo con el proceso de reglamentación de las relaciones internacionales, acentuando su carácter de poder civil transnacional ${ }^{5}$.

\section{Acentuando su carácter de poder civil transnacional, le ue podría participar en la reglamentación de las relaciones internacionales.}

En razón de las diversas corrientes teóricas y normativas, la definición del rol internacional de Europa confronta con numerosos problemas de conceptualización. Como señala Chistopher Hill, remitiéndose al trabajo de Gunnar Sjöstedt acerca de los criterios acerca de la calidad de actor ${ }^{6}$, la consideración de ésta, junto con el criterio de 'presencia' 7 introducido por David Allen y Michael Smith, representaron dos interesantes puntos de partida para la discusión de los últimos años. En este marco, un aspecto central del debate fue la brecha entre las capacidades reales de la UE y las expectativas en cuanto a su accionar ${ }^{8}$. Al respecto, Hill postula la posibilidad de superar el así denominado vacío entre la capacidad y las expectativas y se pronuncia a favor de una interpretación de la UE/EC' ${ }^{9}$ como 'sistema de relaciones exteriores': implícitamente se sugiere tanto que las naciones europeas conforman un subsistema del sistema internacional como tal, como que constituyen un sistema (esto es, no un actor uniforme) que dispone de un amplio espectro de relaciones internacionales (de naturaleza colectiva o individual, así como de orden político y económico) ${ }^{10}$. Más concretamente, dada la brecha entre 'prescripción' y 'realidad' respecto del rol de Europa, Allen y Smith señalan que no puede entenderse a Europa occi-

${ }^{5}$ Véase: Klaus Schubert / Gisela Müller-Brandeck-Bocquet. "Die Europäische Union als Akteur der Weltpolitik: Ein Resümee", en Klaus Schubert / Gisela Müller-Brandeck-Bocquet (Hrsg.). Die Europäische Union als Akteur der Weltpolitik, Leske+Budrich, Opladen, 2000, pág. 288.

${ }^{6}$ De acuerdo con Sjöstedt, un actor internacional constituye una entidad que satisface tres criterios, esto es: está claramente delimitado respecto de otros actores y de su medio ambiente (delimited), es autónomo (autonomous) en el sentido de que dicta sus propias leyes y toma en forma independiente sus propias decisiones, disponiendo además de determinadas condiciones estructurales para el accionar a nivel internacional, como por ejemplo personalidad jurídica, agentes diplomáticos propios, etc. Véase al respecto Gunnar Sjöstedt. "The External Role of the European Union”, Swedish Studies in Internacional Relations, Bd. 7, Westmead, 1997.

${ }^{7}$ Véase David Allen / Michael Smith, 1991, "Western Europe's Presence in the Contemporary International Arena", en Martin Holland (Ed.). The Future of European Political Cooperation. Essays on Theory and Practice, Macmillan, Houndsmills, págs. 97-98. Los autores argumentan que el punto relevante en este contexto no es el actor como tal, sino su 'presencia' en el escenario internacional. Dicha 'presencia' constituye una característica en determinadas áreas de política, que influye en las acciones y expectativas de los participantes, pudiendo ser tanto de naturaleza tangible como intangible.

${ }^{8}$ Véase Christopher Hill. "The Capability-Expectations Gap, or Conceptualizing Europe's International Role”, en Journal of Common Market Studies, Vol. 31, N 3, septiembre 1998, págs. 305-328.

${ }^{9}$ EC: European Community.

${ }^{10}$ Véase Christopher Hill. 1993, op. cit., pág. 322. 
dental como un actor plenamente desarrollado, comparable con el modelo del Estado nacional. Sin embargo, Europa no representa para dichos autores un fenómeno independiente en el ámbito internacional, sino que materializa una 'presencia variable y multidimensional', que juega un rol más o menos activo en los diferentes campos de la política internacional. De esta forma, la presencia internacional de Europa se caracterizaría por su variación y fluctuación, lo que permitiría conceptualizarla como una 'geometría variable' ${ }^{11}$.

\section{La parálisis política exterior europea estaría programada.}

La ya mencionada multidimensionalidad de las relaciones exteriores de la UE con su dualismo implícito (por un lado, las políticas exteriores nacionales involucradas en los procesos de decisiones intergubernamentales del Consejo de la UE, y por el otro, la dimensión comunitaria, supranacional de política exterior), así como la separación entre los aspectos económicos de las relaciones exteriores de la UE bajo el así llamado primer pilar comunitario (organizado en tornos a criterios de supranacionalidad) y los aspec- tos políticos del segundo pilar de las políticas exterior y de defensa comunitarias son aspectos que en la bibliografía tienden a ser visualizados como especialmente problemáticos, que resultarían de la posición independiente de la Comisión Europea y de sus competencias concomitantes en el área de política exterior fijadas en los tratados comunitarios ${ }^{12}$. A raíz de la dinámica particular de los entrelazamientos institucionales, así como de la fragmentación de las relaciones exteriores en diversos pilares, se postula que la parálisis de la política exterior europea estaría preprogramada ${ }^{13}$. A esto se sumaría el 'carácter inconcluso' de la UE como actor internacional, determinado por la falta de una dimensión militar independiente de la Organización del Tratado del Atlántico Norte (OTAN) que pudiera complementar en forma satisfactoria las relaciones exteriores de la UE en sus dimensiones diplomática y económica. Pese al fin de la Guerra Fría y a las enmiendas al Tratado de Ámsterdam, se carecería de una identidad europea común, instancia que posibilitaría una renuncia significativa a la soberanía estatal en favor de instituciones centralizadas en el área de las políticas exterior y de defensa comunitaria: En esta dimensión la cooperación europea seguiría siendo limitada, frag-

${ }^{11}$ Véase David Allen / Michael Smith, 1991, op. cit., págs. 96-104. En este contexto, los autores subrayan el hecho de que la presencia internacional de la UE/EC no puede considerarse equivalente a la presencia internacional de Europa Occidental en su conjunto.

${ }^{12}$ Véase, entre otros, Uwe Schmalz. "Aufbruch zu neuer Handlunsgfähigkeit: Die Gemeinsame Außen- und Sicherheits- und Verteidigungspolitik unter deutscher Ratpräsidenschaft", en Integration 22, 1999, págs. 191-204.

${ }^{13}$ Véase Ralf Roloff. "Die Außenbeziehungen der Europäischen Union zwischen Globalisierung und Regionalisierung”, en Zeitschrift für Politikwissenschaft, 11, Jg., Heft 2, 2001a, pág. 1058. 
mentada e intergubernamental ${ }^{14}$. Aun después de que en la Conferencia de Niza se decidiera el traslado de importantes funciones de la Unión Europea Occidental $(\mathrm{UEO})^{15}$ a la UE, y pese al anclaje explícito de la política europea común de seguridad y defensa en el Tratado de la Unión, no podría hablarse más que de un moderado impulso reformador en este área de política ${ }^{16}$.

\section{Los tres pilares de la Unión Europea} de acuerdo al Tratado de Maastricht

La fragmentación o falta de cabalidad del perfil exterior de la UE no implican en la práctica restricciones automáticas a su accionar. A pesar de la confusión que genera con frecuencia la multidimensionalidad de las relaciones exteriores de la UE constituye para otros autores un rasgo positivo, dado que justamente esa característica podría ser interpretada como un potencial importante para un nuevo tipo de política exterior sumamente prometedor, aun cuando determine un tipo de perfil 'multifacético' ${ }^{17}$. El carácter extraordinario del actor UE se vincularía con la capacidad especial de su accionar con vistas al futuro, dado que la posibilidad de actuar en forma flexible en diferentes niveles permite recurrir al postulado de la subsidiaridad. De esta forma, se podría reaccionar en forma conveniente a diferentes desafíos ${ }^{18}$. En vista de su 'carácter posmoderno' ${ }^{19}$ la UE estaría en condiciones de enfrentar los desafíos impuestos por la crecientemente compleja interdependencia ${ }^{20}$. Incluso algunos autores identificados con la perspectiva neo-realista, las mencionadas cualidades se consideran positivas: en condiciones de un creciente interrelacionamiento global e internacional la estructura de las relaciones exteriores de la UE con sus componentes

${ }^{14}$ Véase Philip Gordon. 1998. "The Limits of Europe's Common Foreign and Security Policy”, en Andrew Moravcsik (Ed.). Centralization of Fragmentation? Europe Facing the Challenge of Deepening, Diversity and Democracy, New York, Council of Foreign Relations, págs. 159-200.

${ }^{15}$ WEU (Western European Union), en su denominación en inglés, institución de defensa europea de la cual son miembros diez Estados miembros de la UE signatarios del Tratado de Bruselas, a los que se suman cinco Estados con estatus de observadores: Dinamarca, Irlanda, Austria, Finlandia y Suecia.

${ }^{16}$ Como subraya Elfriede Regelsberger, la exigencia de unanimidad para la toma de decisiones en el área de la política europea común de seguridad y defensa es, entre otros factores, ejemplo de su carácter aún predominantemente intergubernamental. Véase Elfriede Regelsberger. "Die Gemeinsame, Außenund Sicherheitspolitik nach 'Nizza' - Begrenzter Reformeifer und außervertragliche Dynamik", en Integration 24, Jg. $\mathrm{N}^{\circ} 2 / 2001$, págs. 156-166.

${ }^{17}$ Véase Gisela Müller-Brandeck-Bocquet. "Die Mehrdimensionalität der EU-Außenbeziehungen", en Klaus Schubert / Gisela Müller-Brandeck-Bocquet (Hrsg.), Die Europäische Union als Akteur der Weltpolitik, Leske+Budrich, Opladen, 2001, págs. 29-30.

${ }^{18}$ Véase Klaus Schubert / Gisela Müller-Brandeck-Bocquet, 2000, op. cit., pág. 284.

${ }^{19}$ Véase John Ruggie. "Territoriality and beyond: problematizing modernity in international relations", en International Organization, Vol. 47, № 1, 1993, pág. 140.

${ }^{20}$ Véase Klaus Schubert. "Auf dem Wege zu neuen Formen der Staatlichkeit und zu einer neuen Qualität von Außenpolitik?”, en Klaus Schubert / Gisela Müller-Brandeck-Bocquet (Hrsg.), Die Europäische Union als Akteur der Weltpolitik, Leske+Budrich, Opladen, 2000, pág. 19. 
supranacionales e intergubernamentales puede considerarse como sumamente promisoria $^{21}$. En general, puede afirmarse que pese al desequilibrio existente entre las actividades del primer y del segundo pilares (esto es, un actor bien establecido a nivel económico en contraposición a un novato nivel político-estratégico) ${ }^{22}$, la UE está en condiciones de presentarse en la arena internacional como un actor externo eficiente.

\section{En la política comercial comunitaria están dadas las condiciones habilitadoras de una identidad común.}

\section{LA POLÍTICA COMERCIAL COMUNITARIA}

Sin lugar a dudas, la política comercial comunitaria constituye el núcleo real del accionar externo de la UE, dado que es en esa área de política donde están dadas las condiciones políticas y jurídicas, y con ello las condiciones habilitadoras de una identidad común, que permiten la construcción progresiva de una comunidad. Al respecto, es importante destacar que las áreas altamente estratégicas de la cooperación económica y la política de concreción de tratados representan áreas de competencia del entramado supranacional del primer pilar de la Comunidad, aun cuando dentro del espectro de actividades externas de dicho pilar sea cada vez más difícil separar claramente los acuerdos económicos de los políticos. Es en este contexto donde gana relevancia el monopolio de la Comisión respecto de propuestas y cursos de acción en este campo, pese a que desde hace algún tiempo es posible observar cierto distanciamiento del principio de competencia exclusiva de dicho organismo en favor de una orientación de la política exterior que vincula en forma más estrecha el primer y segundo pilares de la $\mathrm{UE}^{23}$.

Sin embargo, el reconocimiento general de la presencia de la UE en la economía internacional no es automáticamente sinónimo de una valoración positiva. Como observan Allen y Smith, la UE se ha convertido con frecuencia en un factor inhibitorio de los procesos de negociación internacional, ya que en repetidas ocasiones asumiría la posición defensiva de alguno de sus miembros ${ }^{24}$. De hecho, durante la época del Acuerdo General de Aranceles Aduaneros y comercio (GATT) la Comunidad Europea en muy raras oca-

${ }^{21}$ Véase Ralf Roloff, 2001a, op. cit., pág. 1045.

${ }^{22}$ Véase David Gompart. “Akteur oder Statist? Die Rolle der Europäischen Union auf der Weltbühne”, en Internationale Politik, 1/2002, pág. 17.

${ }^{23}$ Véase Gisela Müller-Brandeck-Bocquet, 2000, op. cit., pág. 37 y 41. Respecto de los instrumentos e instituciones de la política de comercio comunitaria, véase Simon Hix. "The political System of the European Union", Palgrave, 1999, págs. 336 y ss.

${ }^{24}$ Véase David Allen / Michael Smith, 1991, op. cit., pág. 117. 
siones se constituyó en mecanismo dinamizador de la liberalización comercial. Por el contrario, en lo tocante a la definición de una política comercial común, los compromisos alcanzados frecuentemente tuvieron tintes antiliberales. A esto se le sumaron las complejas estructuras decisorias ya mencionadas en el sistema multidimensional de la UE, dado que los modos de relacionamiento institucional determinaron muchas veces resultados subóptimos, de escasa viabilidad. Además, hasta la creación de la Organización Mundial del Comercio (OMC), la Comunidad no pudo o no quiso asumir un rol directivo en el régimen de comercio internacional que correspondiera a su potencial económico, por lo que su comportamiento no contribuyó a estabilizar activamente dicho régimen ${ }^{25}$. Aunque desde entonces es posible identificar algunas tendencias positivas ${ }^{26}$, el proteccionismo europeo continúa siendo objeto de críticas ampliamente justificadas. Con respecto al futuro, aún no es posible discernir claramente si el nuevo rol directivo que la UE aspira ejercer en la $\mathrm{OMC}^{27}$ logrará erradicar los viejos reflejos defensivos.

Sin embargo, esto no modifica el hecho de que, gracias a este poder económico que puede considerarse como 'softpower', Europa haya logrado convertirse en actor central de la economía mundial, logrando imponer efectivamente sus intereses y valores. En ese sentido, especialmente tras el fin de la Guerra Fría los canales interregionales ofrecen un elemento adecuado para alcanzar esos fines, pues dadas las cada vez menores capacidades regulatorias de los Estados nacionales, el interregionalismo pasa a ser una instancia clave tanto para la generación de nuevos espacios de acción como para la estabilización de las relaciones internacionales.

\section{El interregionalismo es instancia clave para generar espacios de acción y estabilizar las relaciones internacionales.}

${ }^{25}$ Véase Reinhard Rode. "Die Rolle der Europäischen Union in der politischen Dimension der wirtschaftlichen Globalisierung - Globalisierung und Regionalisierung", en Peter-Christian Müller-Graff (Hrsg.). Die Europäische Gemeinschaft in der Welthandelsorganisation. Globalisierung und Weltmarktrecht als Herausforderung für Europa, Nomos Verlagsgesellschaft, Baden-Baden, 2000, pág. 49.

${ }^{26}$ Entre otras cosas, puede considerarse positivo el hecho de que la UE cada vez recibiera mejores juicios de apreciación en los informes de la OMC en lo que respecta a la no-discriminación y respeto de las reglas multilaterales. A esto se suma la influencia liberalizante que la UE ejerce sobre sus socios comerciales menores, así como los grandes avances hacen la liberalización de los servicios y la presión reformadora a raíz de la implementación del euro. Véase al respecto Rolf Langhammer. "Die Europäische Gemeinschaft in der Welthandelsorganisation. Globalisierung und Weltmarktrecht als Herausforderung für Europa”, Nomos Verlagsgesellschaft, Baden-Baden, 2000, págs. 36-37.

${ }^{27}$ Respecto del nuevo rol de Europa en la OIC véase Pascal Lamy. "Neustart nach Seattle. Europa sucht eine Führungsrolle in der WTO”, en Internationale Politik, 4/20000, págs. 19-26. 
3. El ACTOR UE FRENTE A SUS SOCIOS LATINOAMERICANOS

Desde los tiempos de la colonización y la conquista, Europa y América Latina se han relacionado mediante una multiplicidad de vínculos. En el curso de los últimos años, y en contraposición a su modo de relacionamiento original que ponía énfasis en los vínculos birregionales, y en virtud de los cuales cada región debía materializar una unidad mientras que Europa intentaba articularse como actor homogéneo, la UE ha reaccionado a la creciente heterogeneidad del subcontinente latinoamericano desarrollando amplios mecanismos que pretenden apuntar a la singularidad de las diversas subregiones. Pese a que probablemente este enfoque sea el más adecuado, se teme que involuntariamente esta estrategia estimule el proceso de desvinculación entre las subregiones o incluso entre sus países. Claro ejemplo de ello son los diálogos que la UE mantiene en forma paralela con el Mercosur y con Chile, pese a que este país sea miembro asociado de dicho bloque. Por otra parte, cabe preguntarse si los mecanismos desarrollados hasta ahora son suficientes para fomentar eficientemente la asociación estratégica postulada entre ambas regiones en la Cumbre de Río de 1999. De hecho, muchas de esas denominaciones pomposas sólo generan expectativas desmedidas, que en definitiva, conducen a desilusiones posteriores, lo que implicaría consecuencias negativas para la credibilidad del actor UE.

A partir de una actitud realista, los países latinoamericanos son conscientes de que no constituyen una prioridad en la agenda de política exterior de la UE. Pese a esto, Europa continúa siendo el socio más importante en lo relativo a la diversificación de sus relaciones frente a la presencia determinante de Estados Unidos. A la inversa, la pregunta respecto a la importancia de América Latina para Europa no admite respuestas genéricas, dado que deben considerarse aquí por lo menos dos aspectos: 1) por un lado, los matices subregionales de la cooperación con la UE, con claro énfasis en las relaciones con Chile, con el Mercosur y con México, en forma contraste con un compromiso menor en Centroamérica, el Caribe y la Comunidad Andina ${ }^{28}$;

\section{El interés de Europa por América Latina se centra más bien en el nivel político y no económico.}

2) la importancia desigual que los socios le otorgan a diferentes aspectos de la cooperación: sin lugar a dudas el interés de Europa respecto de América Latina se centra no tanto en la dimensión económica de la relación, sino en el nivel político. Mientras que los europeos subrayan la importancia de cuestiones tales como la la lucha contra el comercio de drogas, la

\footnotetext{
${ }^{28}$ Véase Susanne Gratius, "Lateinamerika und Europa vor dem Gipfeltreffen in Madrid: Interessen, Konflikte und Erwartungen", Europa América Latina: Analysen und Berichte, Nr. 6, marzo de 2002, Konrad Adenauer Stiftung, Rio de Janeiro, pág. 33.
} 
protección del medio ambiente, el fomento de las reformas estructurales, de la democracia y de los procesos regionales de integración, los latinoamericanos enfatizan temas tales como la transferencia de capital, el comercio y la asistencia para el desarrollo. Como tendencia general, puede señalarse que los temas conflictivos (como la transferencia de tecnología, los subsidios agrícolas, los obstáculos al comercio) son trasladados paulatinamente del nivel de diálogo general UE-América Latina a las instancias subregionales.

En lo que hace al eje estratégico para América Latina, esto es, las relaciones económicas, cabe señalar que durante la última década se registró una clara reducción de importancia de Europa en América Latina en la dimensión comercial. Mientras que en los años noventa Europa todavía representaba un tercio de las exportaciones e importaciones de América Latina, su participación actual es de un quinto de aquéllas. Durante el año 2000, la UE exportó mercancías por valor de 54,5 millones de euros, principalmente maquinaria, equipos, instalaciones de diverso tipo y productos químicos. Ese año, el valor de las importaciones europeas desde América Latina alcanzó a 48,8 millones de euros, en los que más del $20 \%$ correspondió a productos agrícolas ${ }^{29}$. En general, América Latina fue el gran perdedor en la relación: mientras que entre 1994 y 1998 las exportaciones europeas hacia el subcontinente latinoamericano crecieron a una tasa promedio del 10,9\%, las importaciones europeas procedentes de América Latina sólo aumentaron un 4,9\%. De esta forma, durante ese período la participación de la UE en las importaciones totales de América Latina se mantuvo constante (más de un 17\%). Por el contrario, la proporción de productos latinoamericanos en las importaciones europeas se redujo de $17,2 \%$ (1994) a $14 \%$ $(1998)^{30}$. En 2000, la participación de América Latina/Caribe en las compras de la UE fue de un $5 \%{ }^{31}$. De esta forma, Europa se sitúa en tercer lugar como mercado de ventas, mientras que sigue ubicándose en el segundo como proveedor, detrás de Estados Unidos. (Ver Tabla al final del artículo).

\section{Europa no es igualmente importante para todos los países latinoamericanos.}

Sin embargo, los datos del comercio interregional requieren un análisis más diferenciado. No todos los países europeos manifiestan interés similar en el vínculo económico con los socios latinoamericanos: mientras que Alemania se ubica en el primer lugar en los aspectos comerciales, España se ha convertido en el primer inversor de la subregión. A ello se suman Italia, Portugal y Gran Bretaña. A la inversa, Europa no es igualmente im-

\footnotetext{
${ }^{29}$ Informaciones obtenidas de www.http//europa.eu.int/comm/world/lac/la.htm.

${ }^{30}$ Informaciones provenientes del banco de datos del IRELA, Daterala, 1999.

${ }^{31}$ Véase Eurostat Pressemitteilung, Nr. 57/2002, 15 de mayo de 2002.
} 
portante para todos los países latinoamericanos. En contraposición al predominio general de los Estados Unidos en el hemisferio occidental, la UE es la contraparte más importante para Chile y para el Mercosur, tanto a nivel de comercio como de inversión.

\section{En la última década se aplicó un enfoque basado en la cooperación descentralizada para fortalecer la sociedad civil.}

Concretamente, entre 1996 y 2000 los flujos de inversión directa de la UE en los países de América Latina y el Caribe se sextuplicaron, es decir, aproximadamente un $12 \%$ de las inversiones directas de la UE se dirigieron a esta región, un tercio de las cuales se radicó en Brasil ${ }^{32}$. (Ver Tabla al final del artículo).

En definitiva, como señala Peter Nunnenkamp, América Latina continúa siendo el principal destino de las inversiones europeas fuera de los Estados de la Organización de Cooperación y Desarrollo Económico (OCDE). Pese a ello, en la competencia global por las inversiones europeas, durante los años noventa la importancia de América Latina se vio menguada tanto en relación con las regiones que gozan de un estatuto comercial privilegiado, como Europa Central, como respecto de regiones excluidas de todo tratamiento preferencial por parte de la UE, como el Asia ${ }^{33}$.

En lo que toca a la cooperación internacional, el actor UE continúa siendo el socio más importante para América Latina, más que Estados Unidos y Japón, que durante los últimos años redujeron claramente su compromiso con la región. En forma genérica, cabe destacar que con el correr del tiempo los objetivos de la cooperación internacional fueron tornándose cada vez más amplios, y pasaron de acuerdos con estricto énfasis económico a tratados que contemplan aspectos como la promoción de la democracia y el respeto de los derechos humanos. Así, aspectos clásicos como el fomento de la industria, la ciencia y la técnica se complementaron con nuevos temas como la protección del medio ambiente y la lucha contra el tráfico de drogas. Especial atención recibió durante los últimos diez años un nuevo enfoque basado en la cooperación descentralizada destinada a fortalecer la sociedad civil, en que se hizo especial hincapié en los intereses de las organizaciones no gubernamentales, las mujeres, los niños, los indígenas, la población marginal que reside en conglomerados urbanos, y en el sector no estructurado.

Sistematizando los diferentes aspectos de la cooperación, puede afirmarse que en lo relativo a la asistencia para el desarro-

${ }^{32}$ Véase Eurostat Pressemitteilung, Nr. 57/2002, 15 de mayo de 2002.

${ }^{33}$ Véase Peter Nunnenkamp. 2001/2002. "Die EU-Osterweiterung als Bedrohung, die Entwicklungspartnerschaft als Chance? - Lateinamerika im regionalen Beziehungsgeflecht der EU”, en Wulfdieter Zippel (Hrsg.). Die Beziehungen zwischen der EU und den Mercosur-Staaten. Stand und Perspektiven, Nomos, Verlagsgesellschaft. Baden-Baden, pág. 142. 
1lo, el apoyo técnico y financiero europeo apuntó a acompañar los esfuerzos de los países latinoamericanos para mejorar la distribución del ingreso, fortalecer el Estado de derecho y el orden democrático y generar mecanismos de desarrollo sustentable. En lo relativo a la cooperación económica, se intenta apuntalar los procesos de integración regional, aumentar la capacidad competitiva de las empresas latinoamericanas en los mercados internacionales y la transferencia de conocimientos técnicos especializados. Así pueden mencionarse programas como AL-INVEST (para fomentar la creación de empresas mixtas de industrias pequeñas y medianas), ALFA (cooperación a nivel universitario), URBAL (fortalecimiento de las relaciones entre ciudades de ambos continentes), ALURE (uso racional de la energía) y @ lis (orientado a superar la brecha digital y desarrollar la sociedad de la información). Finalmente, en lo que hace a ayuda humanitaria, se apunta a apoyar a grupos afectados por catástrofes naturales o desplazados de sus lugares de origen.

\section{El informe Salamanca exige la adopción de una política decidida de cooperación al desarrollo.}

Pese a los múltiples efectos positivos de los programas mencionados, no puede dejar de registrarse que América Latina ocupa el último lugar en la clasificación de destinatarios de la ayuda europea: de un total de 9.300 millones de euros del presupuesto 2000 de la Comisión Europea destinados a la asistencia para el desarrollo, América Latina recibió 429 millones, esto es, un $4 \%{ }^{34}$. A la desproporción de dicho monto frente a las necesidades reales del subcontinente latinoamericano, se suman frecuentes dificultades en el desembolso de los fondos comprometidos para los proyectos en curso. Las debilidades del actor UE en esta materia deben sin embargo encuadrarse en un marco internacional generalmente poco propicio a las iniciativas en el campo del desarrollo. Al respecto, las dificultades en la búsqueda de una solución de transacción satisfactoria en la Cumbre de las Naciones Unidas, realizada en Monterrey en marzo de 2002, fueron en ese sentido sintomáticas. El anuncio de la decisión de la UE de elevar su aporte de un 0,33 a un $0,39 \%$ de su producto interno bruto no representa precisamente una señal revolucionaria, si bien la presentación de un plan de acercamiento gradual a la meta del $0,70 \%$ por parte de los europeos puede considerarse como un buen augurio, ${ }^{35} \mathrm{a}$ lo que hay que sumar el Informe Salamanca presentado al Parlamento Europeo, que exige la adopción de una decidida política de cooperación al desarrollo ${ }^{36}$.

${ }^{34}$ Véase Susanne Gratius, marzo de 2002, op. cit., pág. 21.

${ }^{35}$ Cabe señalar que ya desde los años ' 70 , los países desarrollados postularon un $0,70 \%$ de su PBI como meta para sus compromisos en el área de asistencia para el desarrollo.

${ }^{36}$ Otros aspectos del Informe dignos de mención son el pronunciamiento a favor de una Estrategia Común que fomente la Asociación con los asociados latinoamericanos, lo que exige la culminación 


\section{La Cumbre de MAdrid}

Los resultados de la II Cumbre Europa-América Latina/Caribe, realizada en Madrid, difícilmente pueden haber sorprendido a los observadores y participantes del proceso interregional. Ya las instancias preparatorias indicaban un serio cuestionamiento de las expectativas de lograr avances significativos, debido a diversos factores. Por el lado europeo, el ritmo de los procesos electorales en un país clave como Francia dio lugar a un clima de incertidumbre en torno a temas centrales como las concesiones de la UE en materia de subsidios agrícolas comportamiento que, como se señalaba en las consideraciones teóricas, se convierte una y otra vez en limitante del accionar colectivo de la Unión. Los desafíos urgentes impuestos por la cada vez más cercana ronda de ampliación de la UE concentraron asimismo gran parte de la energía político-diplomática del bloque e impusieron consideraciones presupuestarias ineludibles. El ya mencionado retroceso de los intercambios comerciales no dejó de ser un aspecto que relativizó el significado asignado a la relación interregional. Por el lado latinoamericano, el agravamiento de la crisis argentina marcó de manera especial el evento, dado que la parte española a cargo de la presidencia de la UE ve muy afectados sus intereses por la profunda caída del país sudameri- cano, que también altera radicalmente los planes de inversión y de comercio de los europeos en toda la región debido al temor de los efectos de arrastre en los países vecinos.

\section{El agravamiento de la crisis argentina marcó de manera especial las deliberaciones de la Cumbre de Madrid.}

A partir de este marco, las circunstancias del encuentro eran claramente diferentes del contexto de la I Cumbre en Río de Janeiro. En términos generales, los latinoamericanos condenaron unánimemente los subsidios agrícolas y el proteccionismo agrícola de la UE, que contradice abiertamente su retórica de liberalización comercial a nivel mundial. En lo relativo a la dimensión política se ratificaron principios de derecho internacional vigentes, entre ellos el compromiso de luchar contra el terrorismo y el narcotráfico. En el área económica, entre otros aspectos se hizo referencia al diferente grado de avance en las negociaciones entre la UE y los diferentes grupos subregionales, así como a los progresos de las iniciativas globales en los rubros comercio, desarrollo sostenible, medio ambiente, financiamiento del desarrollo, reforma del sistema financiero, y se prestó apoyo a los esfuerzos de Argentina por lle-

de la red de acuerdos en que se negocian con el Mercosur y con Chile, el establecimiento de un Plan Global de lucha contra la droga, el respeto a las comunidades indígenas y la preservación del medio ambiente. 
gar a un acuerdo con las instituciones financieras internacionales. En el área cultural, se recomendaron un plan de acción en el área de educación superior, un análisis del tema migratorio, la adopción de programas de prevención del Sida y de mitigación de catástrofes naturales. Comentario especial merece la crítica encubierta que las partes lanzaron al 'tercero invisible' en la relación, Estados Unidos: aludiendo a la Ley Helms-Burton y a las nuevas medidas proteccionistas estadounidenses en los rubros del acero y del agro, se rechazaron todas las medidas de carácter unilateral y efecto extraterritorial que son contrarias al derecho internacional y a las reglas de libre comercio aceptadas. A esto se sumó el apoyo expreso a la creación inminente y al funcionamiento efectivo de la Corte Penal Internacional y a la ratificación del Protocolo de Kyoto $^{37}$.

\section{La Ley Helms-Burton fue el "tercero invisible" de la reunión.}

Por otra parte, los resultados alcanzados en el marco de minicumbres con los socios subregionales confirman la diversidad ya mencionada del grado de compromiso con ella:

a) Chile: Sin lugar a dudas, el acontecimiento más destacado fue el anuncio de la conclusión del acuerdo bilateral UEChile, "de cuarta generación plus", que fue dado a conocer a fines de abril ${ }^{38}$. Los compromisos asumidos van más allá de las obligaciones adoptadas en el marco de la OMC, e incluyen un acuerdo de libre comercio de bienes en todos los sectores, un acuerdo relativo a vinos y bebidas alcohólicas, un acuerdo sanitario y fitosanitario, un amplio programa de facilitación del comercio en materia aduanera, un acuerdo de servicios, un acuerdo de inversión, un acuerdo en materia de compras del sector público, así como la definición de reglas de competencia, de propiedad intelectual y de un sistema de solución de controversias. Asimismo, se celebraron convenios sobre diálogo político y sobre cooperación. La firma del acuerdo de libre comercio, que liberalizará más del $90 \%$ de los intercambios bilaterales de bienes en un plazo inferior a diez años, se inserta en una tendencia creciente en las corrientes de comercio, que se elevó de 6.140 millones de euros en 1999 a 8.573 millones en 2000, año en el que la UE importó mercancías chilenas por un valor de $5.113 \mathrm{mi}-$ llones (principalmente productos manufacturados, productos agrícolas no elaborados, bebidas y tabaco). Por su parte, las exportaciones europeas ascendieron a 3.460 millones de euros (sobre todo maquinaria, equipo de transporte, productos químicos y productos manufacturados). En el renglón de inversiones, en 1999 las de la UE se elevaron a 10.412 millones de euros, destacándose los sectores de servicios financieros, construcción y elec-

${ }^{37}$ Véase European Union-Latin America and Caribbean Summit, Madrid, 17 de mayo de 2002, Political Declaration. The Madrid Commitment, 8802/02 (Presse 133): http://ue.eu.int/Newsroom.

${ }^{38}$ Véase EU Institutions Press Releases. Statement of Commissioner Lamy announcing successful end to negotiations on EU-Chile Association Agreement, DN: IP/02/630. Date: 26 de abril de 2002. 
tricidad $^{39}$. En materia política, la declaración conjunta destacó asimismo la coincidencia de las partes en la promoción de los valores democráticos, el Estado de Derecho, el respeto de los derechos humanos y la libertad individual, la aspiración a cooperar en el campo de la política exterior y de la seguridad, así como en la lucha contra el terrorismo, mediante la institucionalización del diálogo político entre el Parlamento Europeo y el Congreso chileno y se destacó el vínculo creciente entre la sociedad civil de ambas partes ${ }^{40}$.

\section{La declaración conjunta destacó la coincidencia de las partes en la promoción de valores democráticos.}

b) Mercosur: la declaración conjunta no deja de subrayar el avance alcanzado por el comité de negociaciones birregionales, destacando el creciente grado de entendimiento alcanzado en el campo político y decidiendo profundizar el diálogo político creando nuevos mecanismos de consulta, como encuentros previos a las conferencias internacionales de las Naciones Unidas o entre las delegaciones acreditadas ante los organismos especializados en Génova, París, Roma, Viena y Nairobi y concretando un acuerdo político sobre defensa de la democracia y cooperación en la lucha contra el terrorismo y el tráfico de drogas. En lo que respecta a cooperación, se apunta a una rápida aplicación del memorádum firmado en Luxemburgo en junio de 2001 que entre otras cosas, abarca temas aduaneros, mercados internos, coordinación de la política macroeconómica, dimensión social del Mercosur, normas y estándares, cuestiones agrícolas, sanitarias y fitosanitarias. En lo que hace a las negociaciones por la Asociación se prevé encuentros a nivel ministerial antes de establecer un futuro comité negociador birregional. Por su parte, se resolvió continuar las conversaciones acerca de la liberalización comercial en un encuentro en julio de 2002 y no se fijó una fecha límite para el cierre de las negociaciones. Otro logro de Madrid fue el lanzamiento de un plan de acción para la facilitación de negocios, que fue apoyado activamente por el Mercosur-EU Business Forum $(\mathrm{MEBF})^{41}$ en una reunión realizada en Buenos Aires en diciembre de 2001 y en la cual se propusieron 62 medidas sobre cuestiones aduaneras, normas, reglamentos y comercio electrónico.

c) Centroamérica: En el marco del diálogo de San José, la XVIII Conferencia ministerial decidió iniciar un programa renovado con líneas de acción prioritarias (democracia, derechos humanos, estado de derecho, gobernabilidad, sociedad civil, integración regional y relaciones económicas interregionales, seguridad, medio ambiente y desastres naturales, consultas so-

\footnotetext{
${ }^{39}$ Véase Bilateral Trade Relations Chile, septiembre de 2001: http://europa.eu.int/comm/trade/bilateral/chl.htm.

${ }^{40}$ Véase Joint Declaration, Madrid, 17 de mayo de 2002. 8874/02 (Presse 142): http://ue.eu.int/ Newsroom.
} 
bre política internacional) al que se dio nuevo formato, previéndose encuentros semestrales entre la Troika de la UE y los ministros de relaciones exteriores centroamericanos $^{42}$. Sin embargo, ello no logró compensar la frustración de los países centroamericanos que aspiraban, al igual que la Comunidad Andina de Naciones, a comenzar a negociar de inmediato un acuerdo similar al que la UE aspira a celebrar con el Mercosur. En definitiva, la fórmula de transacción aprobada finalmente prevé abrir la vía de negociación de un acuerdo de libre comercio y asociación después de 2004 , cuando probablemente ya hayan entrado en vigor los compromisos negociados en el marco de la OMC.

d) Comunidad Andina de Naciones: más allá de la aspiración de celebrar un acuerdo sobre los aspectos mencionados, pese a su brevedad el comunicado conjunto refleja el estancamiento de la relación entre ambos bloques, lo que genera agudas críticas a la presunta indiferencia de la UE, la falta de iniciativas andinas y los escasos resultados concretos de los proyectos conjuntos. Así tras el primer acuerdo de cooperación de 1983 y su replanteamiento mediante el acuerdo de ju- nio de 1992, las últimas reuniones de seguimiento, celebradas en febrero de 1999 en Cartagena de Indias y en diciembre de 2001 en Bruselas, revelan que la insatisfacción de ambas partes es innegable: la Comunidad Andina no ve atendidos sus principales reclamos (acuerdo de asociación, modificación de las decisiones unilaterales de la UE respecto de la aplicación del Sistema Generalizado de Preferencias), mientras que la UE no se siente satisfecha, entre otras cosas, con los resultados del diálogo sobre la fiscalización de precursores químicos ${ }^{43}$. Sin embargo, durante la reunión de Madrid se enfatizaron el fortalecimiento de la democracia en la región andina a partir de la aplicación de la Carta Democrática Interamericana y del proceso de integración regional, subrayándose la necesidad de coordinación en la lucha contra el terrorismo, el tráfico de drogas y los crímenes co$\operatorname{nexos}^{44}$.

\section{Se comprobó el estancamiento de la relación entre la $\mathrm{UE}$ y la Comunidad Andina.}

${ }^{41}$ Véase Second Meeting of Heads of State and of Government of the European Union and of Mercosur, Madrid, 17 de mayo de 2002, Joint Communique, 8803/02 (Presse 134): http://ue.eu.int/ Newsroom.

${ }^{42}$ Véase XVIIth Ministerial Conference of the San José Dialogue, Madrid, 18 de mayo de 2002. Joint Communique, 8806/02 (Presse 137): http://ue.eu.int/Newsroom.

${ }^{43}$ Véase Gianpiero Leoncini. "CAN-UE: ¿Hacia un acuerdo de asociación? La evolución, el estado actual y las perspectivas de las relaciones de la Comunidad Andina con la Unión Europea”, Documentos sobre Integración Andina, http://www.comunidadandina.org.documentos/docIA7IA21-3-02.htm, marzo de 2002.

${ }^{44}$ Véase Meeting between the Heads of State and Governement of the Andean Community of Nations and the European Union, Madrid, 18 de mayo de 2002, Joint Communique. 8804/02 (Presse 135): http:/ /ue.eu.int/Newsroom. 
e) México: por primera vez se concretó en Madrid una cumbre mexicano-europea en el marco del acuerdo de asociación económica, coordinación y cooperación política, firmado en 1997, que desde su entrada en vigencia, en 2000, ha dado lugar a un aumento del $28,6 \%$ en el comercio bilateral, de $44,1 \%$ de las exportaciones mexicanas y de un $23,1 \%$ en las europeas. Se dejó constancia con satisfación del lanzamiento de la iniciativa MexicoEU Business Forum, conviniéndose en la necesidad de profundizar el diálogo político y la cooperación en áreas como ciencia, tecnología, medio ambiente y educación. Además del intercambio de opiniones sobre cuestiones de orden regional e internacional, el presidente Fox dio a conocer los avances del Plan Puebla, en especial en lo relacionado a desarrollo humano, lucha contra la pobreza, promoción de las inversiones y desarrollo productivo en el sur de Mexico y en los países de América Central. Finalmente, México fue designado sede de la tercera Cumbre UEAmérica Latina/Caribe para $2004^{45}$.

\section{REFLEXIONES FINALES}

Sobre la base de la evidencia empírica y yendo más allá de las declaraciones formales de la Cumbre de Madrid, cabe plantearse cuáles son el sentido y el ver- dadero alcance de la supuesta asociación estratégica que se aspira en la relación interregional. En vista del alcance global de diversas áreas temáticas, algunos expertos han postulado la necesidad de conformar una nueva cultura de la cooperación a nivel mundial, no orientada exclusivamente por consideraciones hegemónicas. En el marco del debate en torno de a nuevo "Bretton Woods", la UE como actor con características de actor a nivel mundial tendría teóricamente gran interés en imponer sus concepciones de una economía de mercado que incluya consideraciones de orden social y ecológico. Para ello necesitaría colaboradores: y es aquí donde América Latina y sus foros subregionales se convertirían en interesantes socios. Lo mismo valdría para cuestiones tales como el cambio climático, los peligros que amenazan la biodiversidad, las corrientes migratorias descontroladas, el terrorismo internacional y el tráfico de drogas $^{46}$. Algunos actores clave como el Alto Representante para la política exterior y de defensa comunitaria Javier Solana van aún más lejos, y postulan que, dado que ambos continentes son socios naturales, la era de la globalización será también la era del reencuentro definitivo de Europa y América Latina, la era de la consolidación de una asociación permanente ${ }^{47}$.

${ }^{45}$ Véase México-European Union Summit, Madrid, 18 de mayo de 2002. Press release. 8805/02 (Presse 136-g): http://ue.eu.int/Newsroom.

${ }^{46}$ Véase Klaus Bodemer. "Auf dem Weg zu einer strategischen Partnerschaft? - Die europäischlateinamerikanischen Beziehungen an der Wende zum 21. Jahhundert”, en Wulfdieter Zippel (Hrsg.). Die Beziehungen zwischen der EU und den Mercosur-Staaten, Stand und Perspektiven, Nomos Verlagsgesellschaft. Baden-Baden, 2001/2002, págs. 106-107.

${ }^{47}$ Véase http://www.lanacion.com.ar/02/05/18/dp_397635.asp. 
A la luz de los resultados concretos, no puede menos que llamar la atención el marcado optimismo de afirmaciones de esta naturaleza. El compromiso europeo con la región no proporciona índices claros de una voluntad unívoca de profundizar los vínculos, aunque los motivos de esa actitud distante suelen ser motivados por actitudes o problemas generados al interior de los estados latinoamericanos. Claro ejemplo de ello es la situación en el Cono Sur. No hay duda alguna de que el proteccionismo en materia agrícola impide que fructifiquen las negociaciones con el Mercosur. Pero la crisis del bloque sudamericano, agudizada por la extrema inestabilidad argentina y los rumores en torno al curso que seguirá la nueva administración de Brasil, se torna asimismo en obstáculo esencial para avamzar a nivel interregional.

\section{La política debe entenderse cada} vez más como policéntrica.

Yendo un paso más allá en el análisis, y en relación con las instancias involucradas en el proceso, no puede dejar de constatarse que el postulado de una cultura de la cooperación global es extremadamente difícil de alcanzar en la forma del proceso de arriba hacia abajo, centralizado en el accionar de los estados nacionales. Al respecto, la discusión en torno a la gobernabilidad a nivel mundial demostró que la política debe entenderse cada vez más como policéntrica, donde es posible identificar otros niveles posibles de regulación. Aun cuando por lo general el debate sobre los conceptos estratégicos de gobernabilidad global apunte a la reforma del sistema de las Naciones Unidas, otros componentes, tales como los procesos regionales y su 'producto derivado', el 'interregionalismo, adquieren creciente importancia para la arquitectura global. La inclusión de este último nivel en un proceso de cooperación y concertación de abajo hacia arriba puede significar un aporte relevante frente a los desafíos a nivel mundial. Pero, más allá de las relaciones intergubernamentales, se impone aquí la inclusión aún más decidida de los actores privados y de la sociedad civil.

El riesgo implícito de las relaciones con los así llamados mercados emergentes y con los países en vías de desarrollo dentro de las condiciones de economía globalizada vigentes ya quedó claramente de manifiesto durante la crisis asiática. La actual crisis en Argentina no ha hecho más que confirmar esta posibilidad, con el agravante de posibles efectos de derrame en la subregión. Sin embargo, a largo plazo, un retroceso y la desvinculación progresiva de Europa del subcontinente latinoamericano no sólo representarían claudicar frente a los intereses hegemónicos de Estados Unidos en la región, cuestionando seriamente sus posibilidades como actor internacionalmente relevante incluso en el campo económico-comercial, eje central de su existencia. Sino que, al mismo tiempo, sería una señal profundamente negativa frente las expectativas depositadas por sus socios latinoamericanos en su calidad de 'poder civilizante' en el complejo escenario de la política internacional a comienzos del siglo XXI. 


\section{AnEXos}

\section{Comercio exterior con los países de América Latina y el Caribe en miles de millones de euros}

\begin{tabular}{|c|c|c|c|c|c|c|c|c|}
\hline & \multicolumn{3}{|c|}{$\begin{array}{l}\text { Importaciones de la } \\
\text { UE }\end{array}$} & \multicolumn{3}{|c|}{$\begin{array}{l}\text { Exportaciones de la } \\
\text { UE }\end{array}$} & \multicolumn{2}{|c|}{$\begin{array}{l}\text { Balanza } \\
\text { comercial }\end{array}$} \\
\hline & 1995 & 2000 & $\begin{array}{c}\text { Crecimiento } \\
\text { anual } \\
\text { promedio }\end{array}$ & 1995 & 2000 & $\begin{array}{c}\text { Crecimiento } \\
\text { anual } \\
\text { promedio }\end{array}$ & 1995 & 2000 \\
\hline México & 3,2 & 7,0 & $17 \%$ & 4,5 & 14,0 & $25 \%$ & 1,3 & 7,0 \\
\hline Chile & 3,2 & 5,1 & $10 \%$ & 2,4 & 3,5 & $8 \%$ & $-0,8$ & $-1,6$ \\
\hline Mercosur & 15,0 & 23,6 & $9 \%$ & 16,9 & 23,9 & $7 \%$ & 1,8 & 0,3 \\
\hline $\begin{array}{l}\text { de los cuales: } \\
\text { B rasil }\end{array}$ & 10,8 & 17,6 & $10 \%$ & 11,4 & 16,6 & $8 \%$ & 0,6 & $-1,0$ \\
\hline Argentina & 3,7 & 5,4 & $8 \%$ & 4,6 & 6,2 & $6 \%$ & 0,9 & 0,7 \\
\hline Com unidad Andina & 6,2 & 7,9 & $5 \%$ & 5,5 & 6,9 & $5 \%$ & $-0,7$ & $-1,0$ \\
\hline América Central & 2,2 & 4,3 & $14 \%$ & 2,0 & 3,7 & $13 \%$ & $-0,2$ & $-0,6$ \\
\hline Caricom & 1,4 & 3,1 & $17 \%$ & 1,8 & 3,6 & $15 \%$ & 0,4 & 0,5 \\
\hline $\begin{array}{l}\text { República } \\
\text { D ominicana }\end{array}$ & 0,2 & 0,3 & $8 \%$ & 0,3 & 1,2 & $28 \%$ & 0,1 & 0,8 \\
\hline Cuba & 0,3 & 0,7 & $15 \%$ & 0,7 & 1,4 & $15 \%$ & 0,4 & 0,7 \\
\hline $\begin{array}{l}\text { América } \\
\text { La tina/Caribe }\end{array}$ & 31,8 & 52,0 & $10 \%$ & 34,1 & 58,2 & $11 \%$ & 2,3 & 6,2 \\
\hline Extra-UE en total & 545,3 & 1033,4 & $14 \%$ & 573,3 & 942,0 & $10 \%$ & 28,0 & $-91,4$ \\
\hline
\end{tabular}

Fuente: Eurostat

Inversiones directas de la UE en los países de América Latina y el Caribe: 1996-2000

\begin{tabular}{|c|c|c|c|c|c|c|}
\hline & \multicolumn{5}{|c|}{$\begin{array}{c}\text { Flujos netos anuales en miles de millones de } \\
\text { euros }\end{array}$} & \multirow{2}{*}{$\begin{array}{c}\text { Crecimiento } \\
\text { anual } \\
\text { promedio }\end{array}$} \\
\hline & 1996 & 1997 & 1998 & 1999 & 2000 & \\
\hline América Latina & 6,1 & 15,6 & 27,2 & 36,1 & 38,5 & $58 \%$ \\
\hline de los cuales: México & 0,5 & 2,7 & 1,2 & 1,4 & 2,1 & $47 \%$ \\
\hline Chile & 0,2 & 1,6 & 1,5 & 4,0 & 0,9 & $51 \%$ \\
\hline Mercosur & 4,0 & 6,4 & 21,2 & 28,3 & 24,5 & $57 \%$ \\
\hline de los cuales: Brasil & 2,6 & 4,3 & 17,7 & 11,5 & 18,9 & $64 \%$ \\
\hline Argentina & 1,4 & 2,0 & 3,4 & 16,7 & 5,4 & $40 \%$ \\
\hline Países ACP del Caribe & 0,1 & 0,4 & 0,8 & 0,5 & 2,0 & $121 \%$ \\
\hline América Latina/ Caribe & 6,2 & 16,0 & 28,0 & 36,6 & 40,5 & $60 \%$ \\
\hline Extra-UE en total & 47,0 & 84,7 & 198,2 & 285,4 & 327,0 & $62 \%$ \\
\hline
\end{tabular}

Fuente: Eurostat 
Inversiones directas de la UE en América Latina y el Caribe al año 1999

\begin{tabular}{|l|r|r|}
\hline & $\begin{array}{c}\text { Miles de millones al } \\
\text { finalizar el año }\end{array}$ & $\begin{array}{c}\text { \% de la inversión } \\
\text { total de la UE* }\end{array}$ \\
\hline América Latina & 136,3 & $11,5 \%$ \\
\hline de los cuales: México & 11,5 & $1,0 \%$ \\
\hline Chile & 12,6 & $1,1 \%$ \\
\hline Mercosur & 87,2 & $7,3 \%$ \\
\hline de los cuales: Brasil & 48,2 & $4,1 \%$ \\
\hline Argentina & 38,1 & $3,2 \%$ \\
\hline Países ACP del Caribe & 3,0 & $0,3 \%$ \\
\hline América LatinaC & 139,3 & $11,7 \%$ \\
\hline Extra-UE en total & 1187,0 & \\
\hline
\end{tabular}

Fuente: Eurostat

* Sin considerar las inversiones intra-UE 